\title{
Uptake and Tolerance of Chemotherapy in Elderly Patients with Small Cell Lung Cancer and Impact on Survival
}

\author{
Stacey Fisher, ${ }^{1}$ Turki M. Al-Fayea, ${ }^{2}$ Marcy Winget, ${ }^{1,3}$ He Gao, ${ }^{1}$ and Charles Butts ${ }^{3,4}$ \\ ${ }^{1}$ School of Public Health, University of Alberta, Edmonton, Alberta, Canada T6G 1C9 \\ ${ }^{2}$ Princess Noorah Oncology Centre, King Abdulaziz Medical City, Jeddah, Saudi Arabia \\ ${ }^{3}$ Cancer Care, Alberta Health Services, Edmonton, Alberta, Canada T5J $3 \mathrm{H} 1$ \\ ${ }^{4}$ Department of Oncology, University of Alberta, Edmonton, Alberta, Canada T6G 1Z2 \\ Correspondence should be addressed to Marcy Winget, marcy.winget@albertahealthservices.ca
}

Received 8 August 2012; Revised 5 October 2012; Accepted 26 October 2012

Academic Editor: Frank Buntinx

Copyright ( $) 2012$ Stacey Fisher et al. This is an open access article distributed under the Creative Commons Attribution License, which permits unrestricted use, distribution, and reproduction in any medium, provided the original work is properly cited.

The treatment of elderly cancer patients is complicated by many factors. We sought to assess the uptake and tolerance of chemotherapy among patients 75 years and older diagnosed with small cell lung cancer (SCLC) in years 2004-2008 in Alberta, Canada, and assess their survival. All patients who met the above criteria and had an oncologist-consult were included. Data were obtained from the Alberta Cancer Registry and chart review. A total of 171 patients were included in the study, 117 (68\%) of whom began chemotherapy. Of those, $52 \%$ completed all cycles, $66 \%$ did not have any dose reductions, and $31 \%$ completed all cycles at the recommended dose. The risk of death for patients who did not complete all cycles of chemotherapy was 2.72 ( $95 \%$ CI: 1.52-4.87) and for those who completed all cycles but with a reduced dose was 1.02 (95\% CI: 0.57-1.82) relative to those who completed chemotherapy at full dose after adjusting for several demographic/clinical factors. Our results suggest that a significant proportion of elderly patients are able to tolerate chemotherapy and receive a survival benefit from it while those who experience toxicity may receive a survival benefit from a reduction in chemotherapy dose as opposed to stopping treatment.

\section{Introduction}

Lung cancer is the leading cause of cancer-related death in developed nations [1]. Small cell lung cancer (SCLC) accounts for $13-20 \%$ of all lung cancer diagnoses and is commonly classified into two stages, limited and extensive, according to the Veteran's Administration Lung Cancer Study Group (VALG) classification system [2]. This system is used because most SCLC patients present at a stage for which surgery is not appropriate, and thus are usually unable to be classified by the more commonly used cancer staging classification system, tumor-node-metastasis (TNM), which requires surgical confirmation to achieve an accurate classification [3].

SCLC is characterized generally by a rapid growth rate, initial sensitivity to chemotherapy and radiation, and early metastasis to regional lymph nodes and/or distant sites [3]. Limited SCLC is generally described as disease limited to one hemithorax, while extensive SCLC is described as disease present in both hemithoraxes and/or metastasized to more distant areas of the body. Those with limited stage SCLC have a better prognosis than those with extensive stage disease. The median survival for limited SCLC patients is 23 months, while those with extensive SCLC have a median survival of $8-12$ months, if treatment is administered [4]. Over $50 \%$ of lung cancer patients in Canada are diagnosed at 70 years of age or older while over $20 \%$ are diagnosed at age 80 years or older [5].

The standard of care for patients with SCLC combined concurrent chemoradiotherapy for those with limited disease and chemotherapy alone for those with extensive disease [6]. The preferred chemotherapy regimen is etoposide plus cisplatin; however, etoposide plus carboplatin is an acceptable alternative for patients who are unable to tolerate cisplatin. Many elderly SCLC patients are not selected to receive chemotherapy, however, for fear of toxicity due to 
their age and the presence of comorbidities $[7,8]$. It is also recommended that patients who have achieved remission or stable disease after the completion of primary treatment receive prophylactic cranial radiation (PCI) to reduce the risk of brain metastases [6]. Additionally, if a patient is not a good candidate for or refuses chemotherapy, they may receive radiation to symptomatic sites.

Evidence-based care for the elderly lung cancer population is lacking due to the underrepresentation of this population in clinical trials [9]. Cancer trials tend to consider 70 years of age as the reference point for being "elderly;" however, there is not a specific minimum age that clearly defines the term [10]. The tendency to exclude elderly patients from cancer treatment clinical trials on the basis of chronological age is largely because older patients are more likely to have serious comorbid conditions and have reduced organ function which can lead to higher drugrelated toxicities [11-14]. Many elderly people, however, do not have any measurable loss of functional capacity and are free from significant medical problems [15]. In Canada, it has been estimated that $45 \%$ of those $75-84$ years of age and $22 \%$ of those 85 years of age and older are in "overall good health" [16]. In addition, studies have shown that age alone is not significantly associated with adverse prognosis $[7,17]$. This suggests that elderly patients should not be excluded from therapeutic opportunities solely on the basis of age $[18,19]$.

The care of elderly patients is, however, often complicated by comorbidities, frailty, and decreased organ function. The purpose of this study was to describe the receipt of chemotherapy provided to elderly patients with SCLC in Alberta, Canada, and assess their chemotherapy tolerance and survival. We also sought to identify the reasons for not recommending chemotherapy and for dose reductions and assess the relationship of patient age in these decisions. In the absence of clear evidence from clinical trials, the analysis of the elderly SCLC population through retrospective population-based studies such as this one helps assess and quantify the value of treating elderly cancer patients with chemotherapy.

\section{Methods}

A retrospective, population-based study was conducted on all residents of Alberta, Canada, diagnosed with SCLC at the age of 75 years or older in years 2004-2008 who had an oncologist-consult. Selection of 75 years was chosen because the median patient age for SCLC in Alberta is about 70 years, and we wanted to focus on the "significant minority" of elderly patients. Furthermore, on basis of our clinical experience, we felt that patients who are 75 years and older are the ones for whom ideal treatment is the least clear; we, therefore, selected 75 years as the age cut-off. The province of Alberta consists of an area of $660,000 \mathrm{~km}^{2}$ and has a population of 3.7 million. Approximately $80 \%$ resides in urban areas [20].

The healthcare system in Alberta is funded and administered publically, as it is throughout all of Canada; standard cancer care such as consultations with specialists and chemotherapy is free to residents. Cancer care is organized and coordinated provincially. Consultations with oncologists, nonsurgical cancer treatment, and other services are provided at cancer care facilities. Prior to receiving chemotherapy, a patient must be referred to one of six cancer facilities in the province to have a consultation with an oncologist with whom treatment options are discussed. Two of these cancer facilities are located in the major cities of Edmonton and Calgary; the remaining four are located in smaller cities. Chemotherapy can be provided through any one of the 17 provincial cancer care facilities.

The Alberta Cancer Registry was used to identify patients 75 years of age or older diagnosed with SCLC (International Classification of Diseases for Oncology (ICD-O-3) [21] topography codes C34.0-C34.9 and morphology codes 8040-8045) in years 2004-2008. Patients were excluded if they were not residents of Alberta, were diagnosed with combined small cell and nonsmall cell lung cancers, or had another cancer diagnosis for which they were receiving treatment. Gender, date of birth, and date of death were also obtained from this source. The Alberta Cancer Registry has been repeatedly recognized by the North American Association of Central Cancer Registries (NAACCR) for its high level of completeness and for the timeliness of its data reporting [22].

A chart review was conducted on all potentially eligible patients identified from the cancer registry to identify those who had an oncologist-consult and to obtain details of the chemotherapy received. All patients who had an oncologistconsult were included in the study. The following data were extracted from patient charts: cancer stage; presence and type of comorbidities; Eastern Cooperative Oncology Group (ECOG) performance status; whether or not chemotherapy was recommended by the oncologist; reasons for not recommending chemotherapy; whether chemotherapy was administered or patient refused reasons patient refused chemotherapy; chemotherapy start date; treatment regimen received; whether the patient received all chemotherapy cycles; number of cycles received if less than the complete amount; reasons for incomplete chemotherapy cycles; whether dose reduction occurred; reasons for dose reduction and changes to the initially recommended chemotherapy regimen. A complete course of chemotherapy was defined as receiving any of the regimens once every three weeks for 4 cycles. Dose reduction was defined as any reduction in the dose of chemotherapy administered to the patient, compared to the recommended dose; dose reduction that occurred at any time during chemotherapy treatment, including the first cycle, was included. Information about other treatments received, such as radiation or second-course treatment, were not collected; however, it is likely that most, if not all, patients with limited stage disease who received chemotherapy also received radiation. Patients with extensive stage disease, however, would only have received radiation to relieve symptoms; such treatment is palliative and would not impact survival.

This study was reviewed and approved by the Alberta Cancer Research Ethics Committee. 
2.1. Data Analysis. Descriptive statistics were calculated to describe the utilization and tolerance of chemotherapy in the SCLC patients who had an oncologist-consult. Exploratory data analyses were performed to determine cut-off values for continuous variables and to assess the relationships of these variables with commencing chemotherapy, dose reductions, and not completing all chemotherapy cycles. Chi-square test or Fisher's exact test (if the expected value of a cell was less than 5) were performed to assess the statistical significance of these associations.

Kaplan-Meier (K-M) curves were generated to compare patient survival by age $(<80$ versus $\geq 80)$ and treatment completeness status. In order to ensure chemotherapy, completeness status was known at the beginning of the time period; the start time (T0) was defined as 12 weeks after the oncologist-consult; all patients were followed to the earlier of their death date or December 31, 2010. The log-rank test and the Wilcoxon test were used to determine statistical differences between the curves. The statistical software $\mathrm{R}$ was used to generate the K-M graphs.

Cox proportional hazard models were used to estimate the effect of treatment status on patient survival, adjusting for ECOG score, disease stage, age at diagnosis, number of comorbidities, and chemotherapy regimen used. As in the K$\mathrm{M}$ graphs, the start time (T0) was defined as 12 weeks after the oncologist-consult date in order to categorize patients properly for chemotherapy completion and dose reduction statuses. The Wald Chi-square test was used to calculate $P$ values for the hazard ratio estimates. All $P$ values are based on two-sided tests. SAS software (version 9.2; SAS Institute, Cary, NC, USA) was used to perform all analyses.

\section{Results}

There were 238 patients aged 75 years or older diagnosed with SCLC in Alberta, Canada, in years 2004 to 2008. Of these, 11 were excluded for the following reasons: 2 were not residents of Alberta; 7 had a diagnosis of combined nonsmall cell and small cell lung cancer; and 2 had another cancer diagnosis for which they were receiving treatment. Of the remaining 227 potentially eligible patients, 171 (75\%) had an oncologist-consult to discuss treatment options and were included in this study. There were 56 patients (25\% of those potentially eligible for this study) who did not have an oncologist-consult. Relative to the patients who had an oncologist-consult, those who did not tend to be older $(46 \%$ were older than 80 years compared to $35 \%$ ). Additionally, almost half of them (46\%) died within two weeks of their diagnosis.

Figure 1 shows a flow chart of the proportion of patients for whom chemotherapy was recommended, began chemotherapy, refused it, received regimen, completed chemotherapy, and who received versus who did not receive chemotherapy cycles at the full dose. Of the patients who had an oncologist-consult, $84 \%$ were recommended chemotherapy and $68 \%$ began chemotherapy (including one patient who commenced chemotherapy despite the fact it is not recommended). The chemotherapy regimens received were cisplatin/etoposide, carboplatin/etoposide, cyclophosphamide/adriamycin/vincristine (CAV), and oral etoposide. The regimens most frequently used were combinations carboplatin/etoposide (47\%) and cisplatin/etoposide (31\%). Of those who began chemotherapy, $52 \%$ completed all cycles, $66 \%$ did not have any dose reductions, and 31\% completed all cycles at the recommended dose. Of those who completed all cycles of chemotherapy, $34 \%$ had limited stage disease.

The relationship between the demographic and clinical characteristics of the patients included in the study and age is shown in Table 1. Just over half of the patients in both age groups were male, and $77 \%$ had extensive disease. A higher percentage of patients 80 years and older had an ECOG score of 3 or 4 compared to those $75-79$ years, $47 \%$ versus $39 \%$, respectively, $P=0.18$. Patients 80 years and older were more likely to have two or more comorbidities than those aged 75$79,62 \%$ versus $48 \%$, respectively.

Table 2 displays the demographic and clinical characteristics of the patients included in the study and the relationship between those characteristics and beginning chemotherapy, having a dose reduction, and incomplete chemotherapy cycles. Of the patients included in the study, $57 \%$ were male, $35 \%$ were 80 years or older, $77 \%$ had extensive disease, $42 \%$ had an ECOG score of 3 or 4 , and $24 \%$ had 3 or more comorbidities. A higher percentage of patients aged 75-79 received chemotherapy than those 80 years or older, $74 \%$ versus $58 \%$, respectively, $P=0.15$. Of those who received chemotherapy, a higher percentage of patients 80 years or older had dose reductions than those $75-79$ years, $46 \%$ versus $29 \%$, respectively, $P=0.09$. Just over half of patients in both age groups, however, received all cycles of chemotherapy. Patients with limited disease were more likely to receive chemotherapy than those with extensive disease ( $87 \%$ versus $63 \%, P=0.02)$; of those who received chemotherapy, 52\% (43 patients) with extensive disease did not complete all chemotherapy cycles compared to $36 \%$ (12 patients) with limited stage disease, $P=0.13$. Similarly, patients with a poor performance status (ECOG 3 or 4) were less likely than those with a good performance status to complete all cycles of chemotherapy, $65 \%$ versus $37 \%, P=0.007$.

Of those who had an oncologist-consult, 28 patients $(16 \%)$ were not recommended chemotherapy, and 27 patients (19\%) for whom chemotherapy was recommended refused it. Oncologists' reasons for not recommending chemotherapy and patients' reasons for refusing it are listed in Table 3. The most common reason oncologists indicated for not recommending chemotherapy was patient performance status (22 of 28 patients). The second most common reason was the presence of comorbidities (16 of 28 patients). Most patients who refused chemotherapy did so due to concerns about toxicity (20 of 27 patients).

Of those who began chemotherapy, $33 \%$ had a dose reduction, and $48 \%$ did not complete all treatment cycles. The most common reason for dose reduction was hematological toxicity (30 of 40 patients), while 10 of 40 patients had a dose reduction due to frailty and performance status (Table 4). Similarly, receipt of an incomplete number of chemotherapy cycles was largely attributable to 


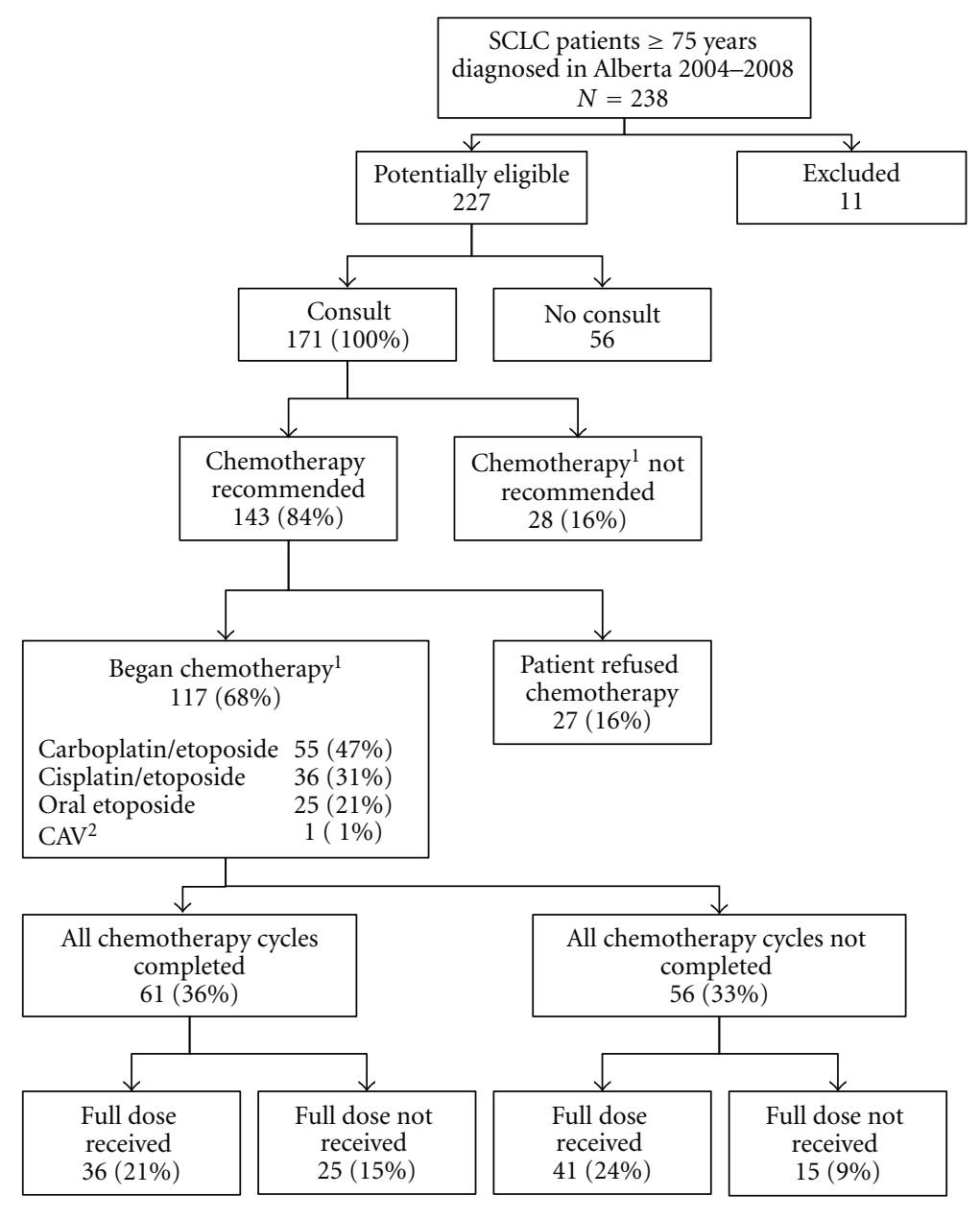

(1) One patient commenced chemotherapy for whom it was not recommended

(2) $\mathrm{CAV}=$ cyclophosphamide, adriamycin, vincristine

FIGURE 1: Flow chart of the number of patients included in the study, had a consult, were recommended chemotherapy, received it, and completed it.

hematological toxicity ( 32 of 56 patients), concerns regarding frailty and performance status ( 25 of 56 patients), and nonhematological toxicity (19 of 56 patients).

Table 5 outlines the drug regimens received and the number of cycles completed by treatment status. Patients who received the carboplatin/etoposide regimen were twice as likely to have dose reductions as those who received oral etoposide, $44 \%$ versus $20 \%$, respectively, $P=0.13$. Conversely, those who received the oral etoposide regimen were more likely to receive an incomplete number of chemotherapy cycles than those who received the combined etoposide/ platin-based regimens, $64 \%$ versus $43 \%$, respectively, $P=$ 0.05 . Of those who began chemotherapy, 61 patients $(52 \%)$ completed all cycles; 25 (41\%) of those who completed all cycles had a dose reduction. A large proportion $(26 \%)$ of people who began chemotherapy only completed one cycle even though $20 \%$ of these people received it at a reduced dose.

All but four patients died by the end of the follow-up period, December 31, 2010. Lung cancer was the recorded cause of death for all patients with the exception of $14 ; 13$ patients died of a noncancer related cause, and one patient died of prostate cancer. Figure 2 displays K-M survival curves by chemotherapy cycle completion status (complete versus incomplete/did not receive) and age group (75-79 versus 80 or older). Those who completed chemotherapy had a better survival rate than those who did not $(P<0.0001)$. The median survival for those who did not complete chemotherapy was 3 months and 23 days, compared to 7 months and 13 days for those who completed all cycles. The survival rate of those who completed chemotherapy did not differ by age group $(P=0.21)$.

Table 6 presents the results from the adjusted survival analysis, generated from the Cox proportional hazards model. Treatment status was the factor most strongly associated with survival. The risk of death for patients who did not complete all cycles of chemotherapy was 2.72 (95\% confidence interval: 1.52 to 4.87 ) relative to those who completed chemotherapy at full dose after adjusting for other variables $(P=0.0007)$. The risk of death for patients who 
TABLE 1: Association of demographic/clinical characteristics and age of patients diagnosed with SCLC who had an oncologist-consult.

\begin{tabular}{|c|c|c|c|}
\hline & & $\begin{array}{l}\text { Age at diagnosis } \\
75-79 \text { years } N(\%)\end{array}$ & $\begin{array}{c}\text { Age at diagnosis } 80 \\
\text { years and older } N(\%)\end{array}$ \\
\hline Total & & 111 & 60 \\
\hline Sex & $P=0.53$ & & \\
\hline Male & & $61(55)$ & $36(60)$ \\
\hline Female & & $50(45)$ & $24(40)$ \\
\hline Stage & $P=1.00$ & & \\
\hline Extensive & & $85(77)$ & $46(77)$ \\
\hline Limited & & $25(23)$ & $13(22)$ \\
\hline Unknown & & $1(1)$ & $1(2)$ \\
\hline ECOG & $P=0.18$ & & \\
\hline 0,1, and 2 & & $51(46)$ & $19(32)$ \\
\hline 3 and 4 & & $43(39)$ & $28(47)$ \\
\hline Missing & & $17(15)$ & $13(22)$ \\
\hline $\begin{array}{l}\text { Number of } \\
\text { co-morbidities }\end{array}$ & $P=0.12$ & & \\
\hline 0 & & $13(12)$ & $3(5)$ \\
\hline 1 & & $44(40)$ & $20(33)$ \\
\hline 2 & & $26(23)$ & $24(40)$ \\
\hline$\geq 3$ & & $28(25)$ & $13(22)$ \\
\hline
\end{tabular}

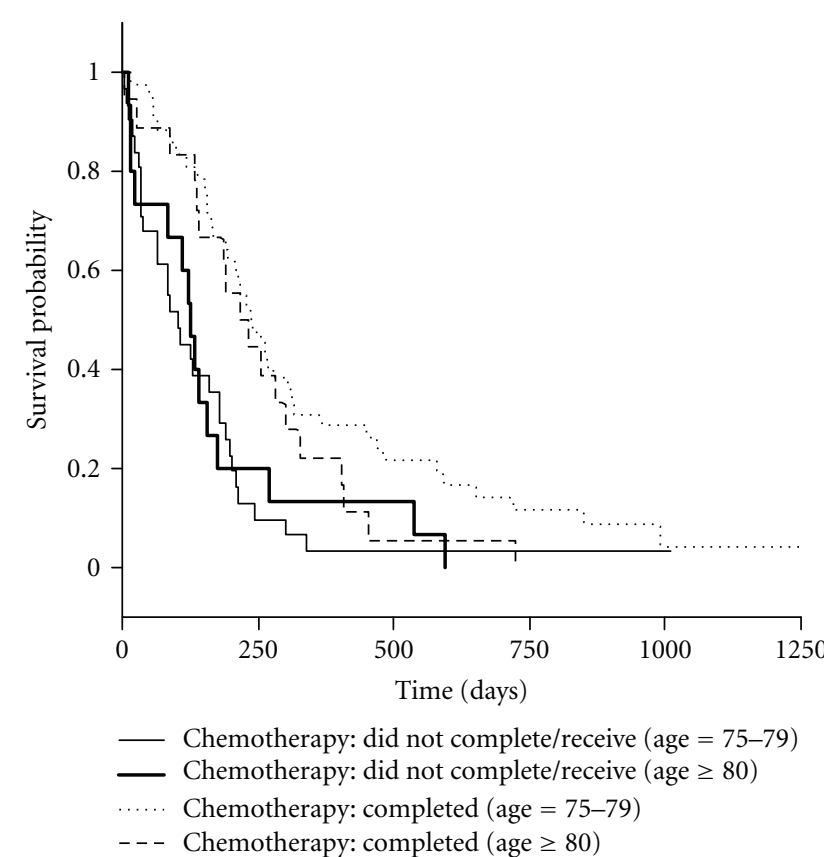

Figure 2: Kaplan-Meier survival curves of all SCLC patients who had an oncologist-consult by chemotherapy completion status, where T0 is 12 weeks after consult.

completed treatment at a reduced dose did not differ from those who completed chemotherapy at the full dose (HR $=1.02,95 \%$ confidence interval: 0.57 to $1.82, P=0.94$ ). Due to the overlapping confidence intervals between the "Completed/reduced dose" group and the "Not completed" group, the model was rerun using the "Completed/reduced dose" group as the reference. In this analysis, the risk of death for patients who did not complete chemotherapy was 2.67 ( $95 \%$ confidence interval: 1.45 to $4.91, P=0.0016$ ) similar to the results shown in Table 6 using the "Completed/full dose" group as the reference.

\section{Discussion}

The purpose of this study was to describe the uptake and tolerance of chemotherapy among elderly patients with SCLC and assess their survival. Thirty-five percent of our study population was aged 80 years and older. These elderly patients, compared to those 75-79 years of age, received less chemotherapy and were more likely to receive a dose reduction, but were equally likely to complete all chemotherapy cycles. Notably, the adjusted hazard ratio of death did not differ between the two age groups. Overall, $52 \%$ of patients who began chemotherapy completed all cycles, and $41 \%$ had reduced chemotherapy doses. These results are confirmation that a significant proportion of elderly patients are able to tolerate chemotherapy and receive a survival benefit from it even in the presence of dose reductions.

Our results also suggest that elderly patients who have their chemotherapy dose reduced but complete all chemotherapy cycles have a similar survival (HR 1.02, CI 0.571.82) to those who complete all chemotherapy cycles at the full dose, after adjusting for ECOG score, disease stage, age, co-morbidity count, and drug regimen. Several phase II clinical trials have tested the efficacy of lower dose combinations of concurrent carboplatin and etoposide regimens in elderly SCLC patients [23-26]. Despite some differences 
TABLE 2: Association of demographic/clinical characteristics and receipt/tolerance of chemotherapy of patients diagnosed with SCLC aging 75 years and older who had an oncologist-consult.

\begin{tabular}{|c|c|c|c|c|}
\hline & $\begin{array}{l}\text { Consult with an oncologist } \\
\qquad N(\%)^{1}\end{array}$ & $\begin{array}{l}\text { Began chemotherapy } \\
\qquad N(\%)^{2}\end{array}$ & $\begin{array}{l}\text { Dose reduction } \\
\qquad N(\%)^{3}\end{array}$ & $\begin{array}{l}\text { Incomplete chemotherapy cycles } \\
\qquad N(\%)^{3}\end{array}$ \\
\hline Total & $171(100)$ & $117(68)$ & $40(34)$ & $56(48)$ \\
\hline Sex & & $P=0.53$ & $P=0.34$ & $P=0.20$ \\
\hline Male & $97(57)$ & $66(68)$ & $25(38)$ & $35(53)$ \\
\hline Female & $74(43)$ & $51(69)$ & $15(29)$ & $21(41)$ \\
\hline Age at diagnosis & & $P=0.15$ & $P=0.09$ & $P=0.76$ \\
\hline 75-79 & $111(65)$ & $82(74)$ & $24(29)$ & $40(49)$ \\
\hline$\geq 80$ & $60(35)$ & $35(58)$ & $16(46)$ & $16(46)$ \\
\hline Year of diagnosis & & $P=0.19$ & $P=0.90$ & $P=0.08$ \\
\hline 2004 & $32(19)$ & $17(53)$ & $6(35)$ & $3(18)$ \\
\hline 2005 & $35(20)$ & $28(80)$ & $8(29)$ & $17(61)$ \\
\hline 2006 & $36(21)$ & $24(67)$ & $9(38)$ & $13(54)$ \\
\hline 2007 & $36(21)$ & $23(64)$ & $7(30)$ & $11(48)$ \\
\hline 2008 & $32(19)$ & $25(78)$ & $10(40)$ & $12(48)$ \\
\hline Stage & & $P=0.02$ & $P=0.78$ & $P=0.13$ \\
\hline Extensive & $131(77)$ & $83(63)$ & $28(33)$ & $43(52)$ \\
\hline Limited & $38(22)$ & $33(87)$ & $12(36)$ & $12(36)$ \\
\hline Unknown & $2(1)$ & $1(50)$ & $0(0)$ & $1(100)$ \\
\hline ECOG & & $P=0.23$ & $P=0.18$ & $P=0.007^{4}$ \\
\hline 0,1 and 2 & $70(40)$ & $59(84)$ & $24(41)$ & $22(37)$ \\
\hline 3 and 4 & $71(42)$ & $40(56)$ & $11(28)$ & $26(65)$ \\
\hline Missing & $30(18)$ & $18(60)$ & $5(28)$ & $8(44)$ \\
\hline Number of co-morbidities & & $P=0.84^{4}$ & $P=0.51^{4}$ & $P=0.89^{4}$ \\
\hline 0 & $16(9)$ & $13(81)$ & $5(38)$ & $7(54)$ \\
\hline 1 & $64(38)$ & $41(64)$ & $16(39)$ & $18(44)$ \\
\hline 2 & $50(29)$ & $30(60)$ & $8(27)$ & $16(53)$ \\
\hline$\geq 3$ & $41(24)$ & $33(80)$ & $11(33)$ & $15(45)$ \\
\hline
\end{tabular}

${ }^{1}$ Column percentage.

${ }^{2}$ Row percentage: denominator is the number who had a consult in corresponding row.

${ }^{3}$ Row percentage: denominator is the number who began chemotherapy in corresponding row.

${ }^{4} P$ values based on Cochran-Armitage test for trend.

TABLE 3: Oncologists' reasons for not recommending chemotherapy and patients' reasons for refusing chemotherapy.

\begin{tabular}{lcc}
\hline & Reasons for not recommending chemotherapy ${ }^{1}$ & Reasons for patients' refusal of chemotherapy $^{1}$ \\
& $N(\%)^{2}$ & $27(100)$ \\
\hline Total & $28(100)$ & $3(11)$ \\
\hline Performance status & $22(79)$ & $4(15)$ \\
Co-morbidities & $16(57)$ & $20(74)$ \\
Toxicity & $0(-)$ & $2(7)$ \\
Lack of social network or support & $1(4)$ & $0(-)$ \\
Wound healing problems & $1(4)$ & $2(7)$ \\
Age & $0(-)$ & $1(4)$ \\
Transportation issues & $0(-)$ & $4(15)$ \\
Other reasons & $0(-)$ & $4(15)$ \\
Unclear & $4(14)$ & \\
\hline
\end{tabular}

${ }^{1}$ The oncologist or patient could have multiple reasons; therefore, the sum of each reason exceeds the total number in each column.

${ }^{2}$ Column percentage. 
TABLE 4: Reasons for dose reduction and not completing chemotherapy.

\begin{tabular}{lcc}
\hline & $\begin{array}{c}\text { Reasons for dose reduction }{ }^{1} \\
N(\%)^{2}\end{array}$ & $\begin{array}{c}\text { Reasons for incomplete chemotherapy cycles }^{1} \\
(\%)^{2}\end{array}$ \\
\hline Total & $40(100)$ & $56(100)$ \\
\hline Hematological toxicity & $30(75)$ & $32(56)$ \\
Nonhematological toxicity & $3(8)$ & $19(34)$ \\
Frailty/performance status & $10(25)$ & $25(44)$ \\
Other medical reason & $5(13)$ & $13(25)$ \\
Patients' decision & $0(-)$ & $2(4)$ \\
Unclear & $6(15)$ & $8(14)$ \\
\hline
\end{tabular}

${ }^{1}$ Multiple reasons are possible; therefore, the sum of the reasons exceeds the total in each column.

${ }^{2}$ Column percentage.

TABLE 5: Drug regimen received and number of cycles completed by treatment status.

\begin{tabular}{lccc}
\hline & Began chemotherapy $N(\%)^{1}$ & Dose reduction $N(\%)^{2}$ & Incomplete chemotherapy cycles $N(\%)^{2}$ \\
\hline Total & $117(100)$ & $40(34)$ & $56(48)$ \\
\hline Drug regimen & & $P=0.13^{3}$ & $P=0.05^{3}$ \\
$\quad$ Carboplatin/etoposide & $55(47)$ & $24(44)$ & $27(49)$ \\
$\quad$ Cisplatin/etoposide & $36(31)$ & $11(31)$ & $12(33)$ \\
oposide & $25(21)$ & $5(20)$ & $16(64)$ \\
CAV & $1(1)$ & $0(0)$ & \\
Numbers of cycles completed & & & \\
1 & $30(26)$ & $6(20)$ & \\
2 & $12(10)$ & $4(33)$ & \\
3 & $14(12)$ & $5(36)$ & \\
$4+^{4}$ & $61(52)$ & $25(41)$ & \\
\hline
\end{tabular}

${ }^{1}$ Column percentage.

${ }^{2}$ Row percentage: denominator is the number who began chemotherapy in corresponding row.

${ }^{3} P$ values based on Fisher's exact test.

${ }^{4}$ Defined as completed chemotherapy cycles.

in treatment schedule and dosing, three of these studies reported similar survival results as the usual treatment regimen, with a median survival of $41-46$ weeks. One of these studies reported lower median survival, however, with a median survival of 37 weeks [26]. Further investigation is, therefore, warranted to better assess the relationship between chemotherapy dose reduction and survival of elderly SCLC patients.

A larger study similar to ours which included a population-based group of elderly SCLC patients was conducted in The Netherlands [27]. The most common reasons for not receiving chemotherapy were a combination of age, co-morbidity, poor performance status, and refusal by the patient or family, which are similar reasons as those identified in our study. They found that $53 \%$ of SCLC patients received chemotherapy, which is also similar to the $52 \%$ of potentially eligible patients from our study that received chemotherapy. As well, a similar percentage of patients had a dose reduction (30\% versus $34 \%$ ) and were unable to complete all chemotherapy cycles ( $43 \%$ versus $47 \%$ ) in The Netherlands study compared to the current study.

Although a large proportion of elderly patients were able to tolerate and experience a survival benefit from chemotherapy, $48 \%$ of patients who began chemotherapy were not able to complete all treatment cycles and did not have a survival benefit. Clearly, all elderly patients are not good candidates for chemotherapy. The difficulty is identifying the ones who are; there is a need for a reliable means to identify elderly patients who would benefit from chemotherapy that does not base its conclusions on chronological age and rather aims to determine biological age by the measurement of objective standard measures $[6,19,28]$. The use of a multidimensional geriatric assessment tool for this purpose has been suggested as a means of achieving this end [19, 29]. Suggested domains of the assessment tool include co-morbidity, functional status, emotional conditions and mental status, social support, polypharmacy, and nutritional state. Even though the tool was first introduced over 15 years ago, the best form of the tool has yet to be defined $[6,29]$. Further efforts are needed to optimize such a tool and implement it in routine practice.

A limitation to the study is the nature of all retrospective studies in that they cannot prove causality. Additionally, there is always selection bias in terms of which patients receive treatment in a real clinical situation as opposed to a clinical trial setting. On the other hand, an inherent strength of a population-level retrospective study such as this one is that the treatment and outcome can be described for every 
TABLE 6: Adjusted ${ }^{1}$ hazard ratio of death of patients 75 years or older diagnosed with SCLC in 2004-2008 in Alberta, Canada, who had an oncologist-consult ${ }^{2}$.

\begin{tabular}{|c|c|c|}
\hline & Adjusted $^{1}$ hazard ratio $(95 \% \mathrm{CI})$ & $P$ value \\
\hline ECOG Score & & $P=0.02$ \\
\hline 0,1 , and 2 & 1 & \\
\hline 3 and 4 & $2.01(1.22,3.31)$ & 0.007 \\
\hline Missing & $1.59(0.88,2.88)$ & 0.12 \\
\hline Stage & & $P=0.33$ \\
\hline Limited & 1 & \\
\hline Extensive & $1.24(0.80,1.92)$ & 0.33 \\
\hline Age at diagnosis & & $P=0.80$ \\
\hline 75-79 & 1 & \\
\hline$\geq 80$ & $1.06(0.66,1.75)$ & 0.80 \\
\hline Co-morbidities & & $P=0.05$ \\
\hline 0 or 1 & 1 & \\
\hline 2 or more & $1.63(1.00,2.66)$ & 0.05 \\
\hline Drug regimen & & $P=0.82$ \\
\hline Cisplatin/etoposide & 1 & \\
\hline Carboplatin/etoposide & $1.15(0.5,2.65)$ & 0.56 \\
\hline Oral etoposide & $1.15(0.71,1.89)$ & 0.75 \\
\hline Treatment status & & $P=0.0018$ \\
\hline Complete/full dose & 1 & \\
\hline Complete/reduced dose & $1.02(0.57,1.82)$ & 0.94 \\
\hline Not completed & $2.72(1.52,4.87)$ & 0.0007 \\
\hline No chemotherapy & $2.01(0.97,4.18)$ & 0.6 \\
\hline
\end{tabular}

${ }^{1}$ Adjusted for all variables shown in the table.

${ }^{2}$ Start time was 12 weeks after the date of the initial oncologist-consult.

patient, as we have done so herein. The major limitations are in obtaining complete information on all the factors that might impact one of or both treatment and outcome in order to properly adjust for them in analyses. In our study we had limited information on the patients who did not have an oncologist-consult, representing 25\% of the entire population of SCLC patients aged 75 years or older in Alberta. The data we do have, however, suggests that many of these patients would not have been candidates for any kind of treatment as they died very soon after being diagnosed. It is possible, however, that some of them could have benefited from chemotherapy but did not have the opportunity because they were not referred to an oncologist, were unable to obtain transportation to an oncologist, were not interested in receiving chemotherapy, or another reason. We were not able to identify reasons for not seeing an oncologist. Regarding the patients who did have an oncologistconsult, performance status was missing for $18 \%$ of patients, and we did not collect information on receipt of other treatment modalities which could have affected survival and the ability to tolerate chemotherapy. It is possible that there is also missing/incomplete information related to the specific reasons for dose reductions and incomplete cycles. A further limitation arises from the relatively small number of patients with limited disease, which prevented us from fully exploring the interrelationships between age, stage, chemotherapy uptake, tolerance, and survival. Further study in a larger patient population may provide interesting insights on these issues.

\section{Conclusion}

SCLC is a significant health issue of the elderly. We have shown that while an appreciable proportion of elderly patients diagnosed with SCLC do not begin chemotherapy treatment, those that do are able to tolerate the treatment and receive survival benefits from it. It is, therefore, vital that elderly patients as well as younger patients are considered for established treatment. Our results also suggest that elderly SCLC patients who complete chemotherapy at a reduced dose have a similar prognosis to those who receive the full dose. Future research should focus on better understanding the relationship between frailty and toxicity to ensure the careful selection of patients who will benefit from chemotherapy treatment.

\section{Authors' Contribution}

S. Fisher and T. M. Al-Fayea contributed equally to this paper. 


\section{Acknowledgments}

The authors thank John Fleming for creating the online data collection tool used for conducting the chart review and also for reviewing the analyses for accuracy. The authors also thank Angela Bella for assisting with formatting and references.

\section{References}

[1] A. Jemal, F. Bray, M. M. Center, J. Ferlay, E. Ward, and D. Forman, "Global cancer statistics," CA Cancer Journal for Clinicians, vol. 61, no. 2, pp. 69-90, 2011.

[2] G. R. Simon and A. Turrisi, "Management of small cell lung cancer: ACCP evidence-based clinical practice guidelines ( $2^{\text {nd }}$ edition)," Chest, vol. 132, supplement 3, pp. S324-S339, 2007.

[3] D. M. Jackman and B. E. Johnson, "Small-cell lung cancer," The Lancet, vol. 366, no. 9494, pp. 1385-1396, 2005.

[4] A. Kurup and N. H. Hanna, "Treatment of small cell lung cancer," Critical Reviews in Oncology/Hematology, vol. 52, no. 2, pp. 117-126, 2004.

[5] Canadian Cancer Society's Steering Committee on Cancer Statistics, Canadian Cancer Statistics 2012, Canadian Cancer Society, Toronto, ON, Canada, 2012.

[6] A. G. Pallis, F. A. Shepherd, D. Lacombe, and C. Gridelli, "Treatment of small-cell lung cancer in elderly patients," Cancer, vol. 116, no. 5, pp. 1192-1200, 2010.

[7] J. J. S. Ludbrook, P. T. Truong, M. V. MacNeil et al., "Do age and comorbidity impact treatment allocation and outcomes in limited stage small-cell lung cancer? A community-based population analysis," International Journal of Radiation Oncology Biology Physics, vol. 55, no. 5, pp. 1321-1330, 2003.

[8] M. L. G. Janssen-Heijnen, R. M. Schipper, P. P. A. Razenberg, M. A. Crommelin, and J. W. W. Coebergh, "Prevalence of comorbidity in lung cancer patients and its relationship with treatment: a population-based study," Lung Cancer, vol. 21, no. 2, pp. 105-113, 1998.

[9] L. F. Hutchins, J. M. Unger, J. J. Crowley, C. A. Coltman, and K. S. Albain, "Underrepresentation of patients 65 years of age or older in cancer- treatment trials," New England Journal of Medicine, vol. 341, no. 27, pp. 2061-2067, 1999.

[10] L. Balducci, "Geriatric oncology: challenges for the new century," European Journal of Cancer, vol. 36, no. 14, pp. 1741$1754,2000$.

[11] K. W. L. Yee, J. L. Pater, L. Pho, B. Zee, and L. L. Siu, "Enrollment of older patients in cancer treatment trials in Canada: why is age a barrier?" Journal of Clinical Oncology, vol. 21, no. 8, pp. 1618-1623, 2003.

[12] D. H. Johnson, "Treatment of the elderly patient with smallcell lung cancer," Chest, vol. 103, no. 1, 1992.

[13] S. M. Lichtman, J. A. Skirvin, and S. Vemulapalli, "Pharmacology of antineoplastic agents in older cancer patients," Critical Reviews in Oncology/Hematology, vol. 46, no. 2, pp. 101-114, 2003.

[14] R. Yancik, R. J. Havlik, M. N. Wesley et al., "Cancer and comorbidity in older patients: a descriptive profile," Annals of Epidemiology, vol. 6, no. 5, pp. 399-412, 1996.

[15] L. Z. Rubenstein, K. R. Josephson, M. Nichol-Seamons, and A. S. Robbins, "Comprehensive health screening of well elderly adults: an analysis of a community program," Journals of Gerontology, vol. 41, no. 3, pp. 342-352, 1986.

[16] M. Shields and L. Martel, "Healthy living among seniors," Health Report, vol. 16, pp. S7-S20, 2006.
[17] M. P. Lebitasy, G. Hédelin, A. Purohit, L. Moreau, F. Klinzig, and E. Quoix, "Progress in the management and outcome of small-cell lung cancer in a French region from 1981 to 1994," British Journal of Cancer, vol. 85, no. 6, pp. 808-815, 2001.

[18] M. Weinmann, B. Jeremic, M. Bamberg, and C. Bokemeyer, "Treatment of lung cancer in elderly part II: small cell lung cancer," Lung Cancer, vol. 40, no. 1, pp. 1-16, 2003.

[19] S. Monfardini, L. Ferrucci, L. Fratino et al., "Validation of a multidimensional evaluation scale for use in elderly cancer patients," Cancer, vol. 77, no. 2, pp. 395-401, 1996.

[20] Statistics Canada, "Population, urban and rural, by province and territory (Alberta table)," Population, urban and rural, by province and territory, Population estimates and projection, Population and democracy, Summary Tables , 2006, last updated September 22, 2009, http://www.isesd.cv.ic.ac.uk/, 2012.

[21] Fritz, C. Percy, A. Jack et al., Eds., International Classification of Diseases For Oncology, World Health Organization, Geneva, Switzerland, 3rd edition, 2000.

[22] T. C. Tucker, H. L. Howe, and H. K. Weir, "Certification for population-based cancer registries," Journal of Registry Management, vol. 26, pp. 24-27, 1999.

[23] K. Shibata, K. Kasahara, Y. Nakatsumi, T. Bando, M. Fuigmura, and T. Matsuda, "Carboplatin plus etoposide in the treatment of small cell lung cancer," Lung Cancer, vol. 18, supplement 1, p. 54, 1997.

[24] K. Matsui, N. Masuda, M. Fukuoka et al., "Phase II trial of carboplatin plus oral etoposide for elderly patients with smallcell lung cancer," British Journal of Cancer, vol. 77, no. 11, pp. 1961-1965, 1998.

[25] H. Okamoto, K. Watanabe, Y. Nishiwaki et al., "Phase II study of area under the plasma-concentration-versus-time curvebased carboplatin plus standard-dose intravenous etoposide in elderly patients with small-cell lung cancer," Journal of Clinical Oncology, vol. 17, no. 11, pp. 3540-3545, 1999.

[26] S. Larive, P. Bombaron, R. Riou et al., "Carboplatin-etoposide combination in small cell lung cancer patients older than 70 years: a phase II trial," Lung Cancer, vol. 35, no. 1, pp. 1-7, 2002.

[27] M. L. G. Janssen-Heijnen, H. A. A. M. Maas, S. A. M. van de Schans, J. W. W. Coebergh, and H. J. M. Groen, "Chemotherapy in elderly small-cell lung cancer patients: yes we can, but should we do it?" Annals of Oncology, vol. 22, no. 4, pp. 821-826, 2011.

[28] M. L. G. Janssen-Heijnen, H. A. A. M. Maas, S. Siesling, C. C. E. Koning, J. W. W. Coebergh, and H. J. M. Groen, "Treatment and survival of patients with small-cell lung cancer: small steps forward, but not for patients > 80," Annals of Oncology, vol. 4, pp. 954-560, 2012.

[29] C. Gridelli, R. De Vivo, and S. Monfardini, "Management of small-cell lung cancer in the elderly," Critical Reviews in Oncology/Hematology, vol. 41, no. 1, pp. 79-88, 2002. 


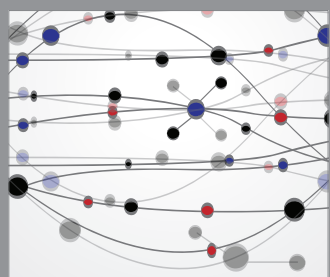

The Scientific World Journal
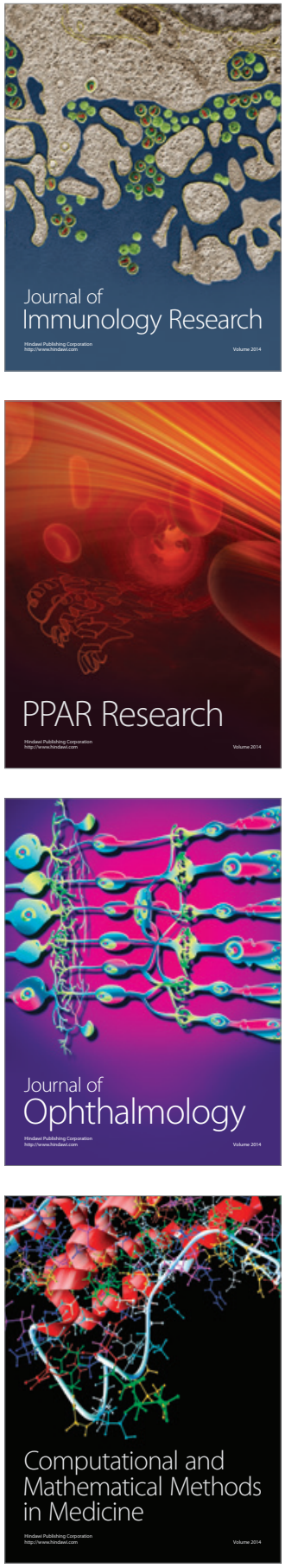

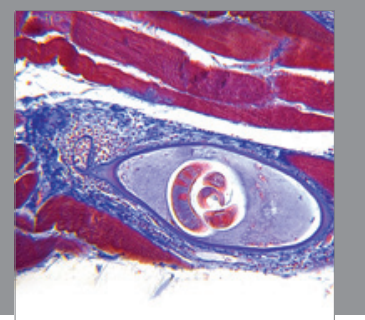

Gastroenterology

Research and Practice
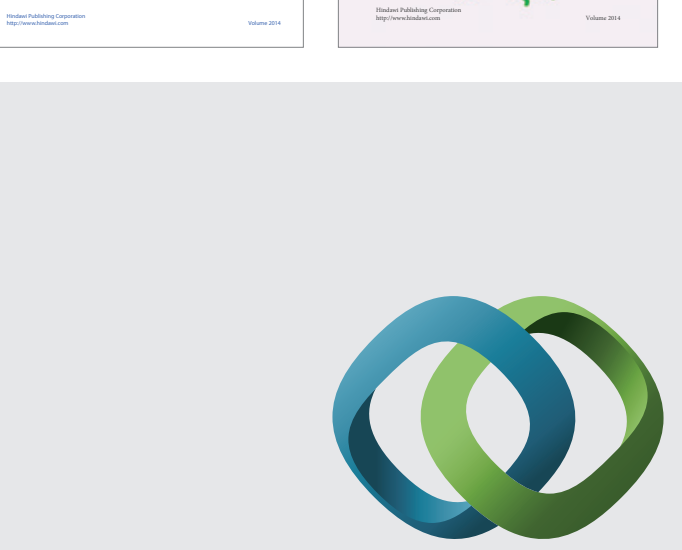

\section{Hindawi}

Submit your manuscripts at

http://www.hindawi.com
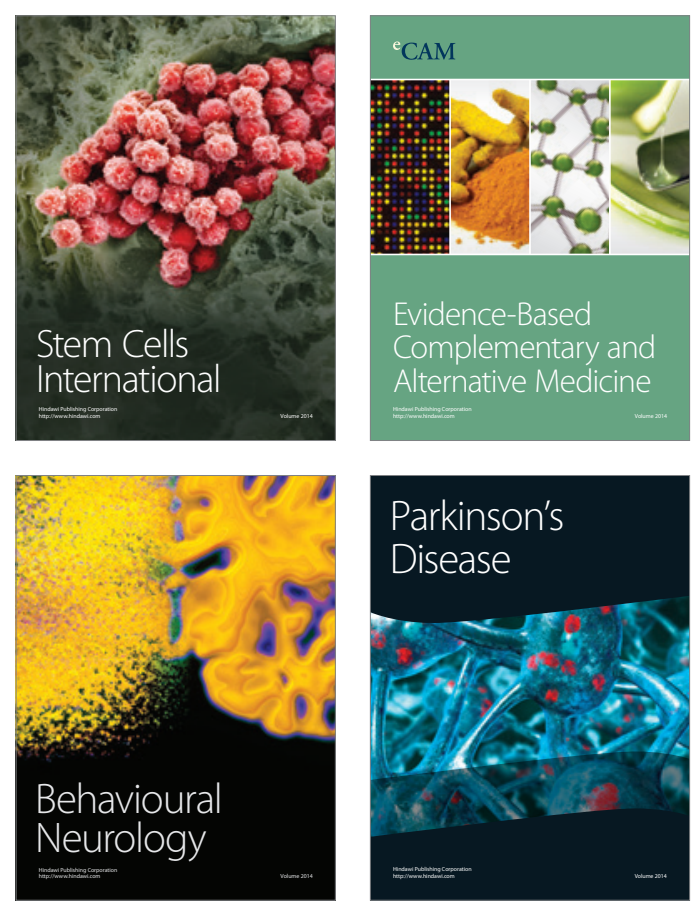

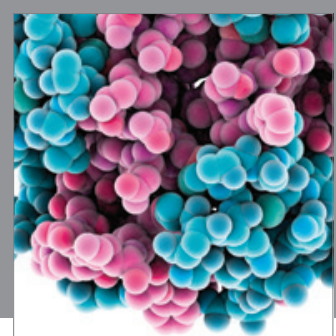

Journal of
Diabetes Research

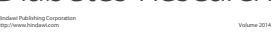

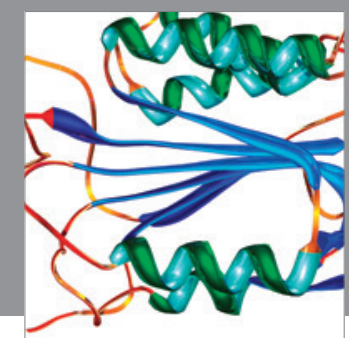

Disease Markers
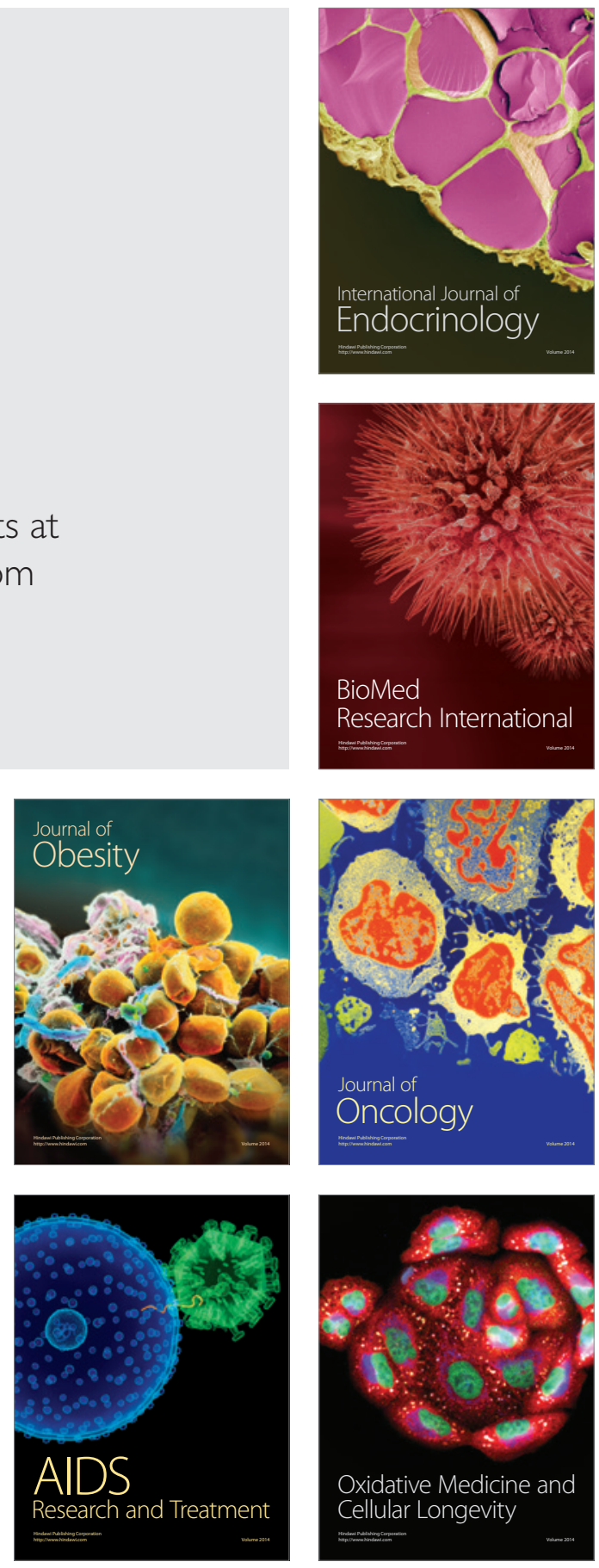